\title{
SINGULAR INTEGRALS AND FRACTIONAL INTEGRALS IN TRIEBEL-LIZORKIN SPACES AND IN WEIGHTED $L^{p}$ SPACES
}

\section{DASHAN FAN * AND HUNG VIET LE}

Abstract. We study the hypersingular integral

$$
T_{h, \alpha} f(x)=\lim _{\epsilon \rightarrow 0} \int_{|y|>\epsilon} \frac{b(|y|) e^{i h(|y|)} \Omega\left(y^{\prime}\right)}{|y|^{n+\alpha}} f(x-y) d y
$$

and the fractional integral

$$
I_{h, \alpha} f(x)=\int_{R^{n}} \frac{b(|y|) e^{i h(|y|)} \Omega\left(y^{\prime}\right)}{|y|^{n-\alpha}} f(x-y) d y
$$

in Triebel-Lizorkin spaces and weighted $L^{p}$ spaces. Here $\Omega \in H^{r}\left(S^{n-1}\right)$, and $b(|y|)$ and $h(|y|)$ are measurable radial functions which satisfy some suitable conditions. We also consider the above integrals along some surfaces of revolution. The results in this paper extend some known results about hypersingular integrals and fractional integrals.

Mathematics subject classification (2000): 42B20, 42B25.

Key words and phrases: Singular integrals, fractional integrals, Triebel-Lizorkin spaces.

\section{REFERENCES}

[1] JÖRAN BERGH, JÖRGEN LÖFSTRÖM, Interpolation Spaces: An Introduction, Springer-Verlag, Berlin Heidelgerg, New York, 1976.

[2] A. P. CALDERón, A. Zygmund, On the existence of certain singular integrals, Acta Math. 88, (1952), 85-139.

[3] A. P. Calderón, A. Zygmund, On singular integrals, Amer. J. Math. 78 (1956), 289-309.

[4] JiECHENG CHEN, DASHAN FAN, YIMING YING, Singular integral operators on function spaces, J. Math. Anal. Appl. 276, (2002), no. 2, 691-708.

[5] JiECheng CHEN, DAShan FAN, YIMING Ying, Certain operators with rough singular kernels, Canad. J. Math., 55 (2003), no. 3, 504-532.

[6] LunG-KeE CHEN, On a singular integral, Studia Math. 85 (1986), no. 1, 61-72 (1987).

[7] LeOnardo Colzani, Hardy Spaces on Sphere, Ph.D. Thesis, Washington University, St. Louis, 1982.

[8] Leonardo Colzani, Mitchell H. Taibleson, Guido Weiss, Maximal estimates for Ces à ro and Riesz means on spheres, Indiana Univ. Math. J., 33 (1984), no. 6, 873-889.

[9] YONG Ding, SHANZHEN Lu, Weighted norm inequalities for fractional integrals operators with rough kernel, Can. J. Math., 50, (1998), no. 1, 29-39.

[10] JAVIER DUOANDIKOETXEA, José L. RUBIO DE FRAnCIA, Maximal and singular integral operators via Fourier transform estimates, Invent. Math. 84 (1986), no. 3, 541-561.

[11] Robert Fefferman, A note on singular integrals, Proc. Amer. Math. Soc., 74 (1979), 266-270.

[12] Hung VIET LE, Hypersingular integral operators along surfaces, Integral Equations Operator Theory 44 (2002), no. 4, 451-465.

[13] Hung VIET LE, Singular integral operators along surfaces of revolution, J. Math. Anal. Appl. 274 (2002), no. 2, 608-625. 
[14] Hung VIET Le, On maximal operators along surfaces. Integral Equations Operator Theory 37 (2000), no. $1,64-71$.

[15] BENJAMIN MUCKENHOUPT, RICHARD L. WHEEDEN, Weighted norm inequalities for fractional integrals, Trans. Amer. Math. Soc., 192, 261-274.

[16] J. NAMAZI, A Singular Integral, Ph.D. Thesis, Indiana University, Bloomington, Indiana, 1984.

[17] Elias M. SteIn, Singular Integrals and Differentiability Properties of Fucntions, Princeton University Press, Princeton, New Jersey, 1970.

[18] Elias M. StEIN, Harmonic Analysis: Real-Variable Methods, Orthogonality, and Oscillatory Integrals, Princeton University Press, 1993.

[19] Elias M. StEIN, GUIDO WeISS, Interpolation of operators with change of measures, Trans. Amer. Math. Soc., 87, (1958), no. 1, 159-172.

[20] HANS TRIEBEL, Theory of Function Spaces, Monographs in Mathematics, 78, Birkhäuser Verlag, Basel, 1983.

[21] HANS TRIEBEL, "Interpolation Theory, Function Spaces, Differential Operators, " (1978) North-Holland Publishing Company, Amsterdam, New York, Oxford. 